\title{
Positive Maternal Attitudes during Infancy and a Positive Lifestyle May Be Associated with Beneficial Epigenetic Alterations and Improved Oral Health
}

\author{
Riichiro Ishida \\ RIKEN (The Ishida-Riichiro Institute), Niigata, Japan \\ Email: ishida-riichiro@yf6.so-net.ne.jp
}

How to cite this paper: Ishida, R. (2019). Positive Maternal Attitudes during Infancy and a Positive Lifestyle May Be Associated with Beneficial Epigenetic Alterations and Improved Oral Health. Psychology, 10, 742-747.

https://doi.org/10.4236/psych.2019.105048

Received: March 30, 2019

Accepted: April 27, 2019

Published: April 30, 2019

Copyright () 2019 by author(s) and Scientific Research Publishing Inc. This work is licensed under the Creative Commons Attribution International License (CC BY 4.0).

http://creativecommons.org/licenses/by/4.0/

\begin{abstract}
Periodontitis causes various diseases, including arterial sclerosis, cardiac infarction, diabetes, aspiration pneumonia, and tooth loss, all of which lower the quality of life of individuals. Risk factors of periodontitis include a lack of oral care and low secretion of saliva due to stress, accompanied by excessive activity of the sympathetic nervous system and low secretion of serotonin and melatonin. Serotonin may improve the ability of individuals to manage stressful events, while melatonin may enhance the regeneration of tooth tissues. Saliva contains melatonin, epidermal growth factor, and nerve growth factor. The production and secretion of serotonin and melanin involve epigenetic mechanisms, i.e., gene expression resulting from chemical responses with genome sequences. Warm-hearted maternal attitudes to babies during infancy and a positive lifestyle, including a well-balanced diet, moderate aerobic exercise, and a purpose in life, as well as warm-hearted human relations, may also improve the ability of individuals to manage stressful events. This improvement in stress management may be linked to beneficial epigenetic alterations that promote the secretion of sufficient amounts of serotonin and melatonin, which may in turn contribute to the prevention and treatment of periodontitis and the regeneration of tooth tissues.
\end{abstract}

\section{Keywords}

Stress Management, Periodontitis, Tooth Tissue Regeneration, Epigenetics, Well-Balanced Diet, Maternal Attitudes, Moderate Aerobic Exercise, Purpose in Life (PIL)/Ikigai 


\section{Introduction}

Periodontitis causes various diseases, including arterial sclerosis, cardiac infarction, diabetes, aspiration pneumonia, and tooth loss, all of which lower the quality of life (QOL) of individuals (Dhadse, Gattani, \& Mishra, 2010; Ferreira, Dias-Pereira, Branco-de-Almeida, Martins, \& Paiva, 2017; Martinez-Herrera, Silvestre-Rangil, \& Silvestre, 2017). Risk factors of periodontitis include smoking, low secretion of saliva, and a lack of oral care, such as insufficient tooth brushing (Sakai, 2016). The autonomic nervous system, which is involved in the secretion of neurotransmitters and hormones, regulates the secretion of saliva (Ishida, 2016; Reece, Urry, Cain, Wasserman, Minorsky, \& Jackson, 2011). Stress, i.e., excessive activity of the sympathetic nervous system, often causes a reduction in the secretion of saliva (Sakai, 2016). Saliva contains important components, such as melatonin, epidermal growth factor (EGF), and nerve growth factor (NGF) (Obana-Koshino, Ono, Miura, Sakai, Uchida, Nakamura et al., 2015). Due to its components, saliva has various functions, including in tooth development (Kabasawa, 2017; Obana-Koshino et al., 2015), bone induction (Kabasawa, 2017), enhancement of swallowing, maintenance of the appropriate balance of oral bacteria, immune function, and tissue repair (Reece, Urry, Cain, Wasserman, Minorsky, \& Jackson, 2011; Sakai, 2016). The term epigenetic refers to gene expression due to chemical responses on deoxyribonucleic acid (DNA) without changes to the genome sequences, e.g., DNA methylation, DNA demethylation, and histone modulation (Reece, Urry, Cain, Wasserman, Minorsky, \& Jackson, 2011; Tajima, 2013). Recently, research in dental medicine has been focused on the functions of serotonin (5-hydroxytryptamine; $\mathrm{C}_{10} \mathrm{H}_{12} \mathrm{~N}_{2} \mathrm{O}$ ), which is a neurotransmitter (Ota, 2013), and melatonin (N-acetyl-5-methoxytryptamine; $\mathrm{C}_{13} \mathrm{H}_{16} \mathrm{~N}_{2} \mathrm{O}_{2}$ ), which is a hormone, from the viewpoint of epigenetics (Obana-Koshino et al., 2015; Yeh, Lin, Yang, Yang, Su, \& Yang, 2016). Importantly and interestingly, epigenetic variations can be inherited and are reversible by external factors and individual experiences (Kubota, 2016). Therefore, changes in lifestyles may contribute to the prevention and treatment of periodontitis and the regeneration of tooth tissues (Kubota, 2016).

Positive maternal attitudes to infants (Ota, 2013) and a positive lifestyle, including a well-balanced diet, moderate aerobic exercise, and a purpose in life (PIL)/ikigai (Ishida, 2014; Ishida, 2016), contribute to improving the ability of individuals to handle stressful events. The term PIL comes from existential philosophy, and the term ikigai is a Japanese word similar to PIL that has been used since ancient times in Japan. The intensity of PIL/ikigai can be measured by the Purpose in Life Test, which is used for psychological evaluations (Sato, Tanaka, Saito, \& Chiba, 1998). PIL/ikigai reflects the function of the prefrontal cortex (PFC), and it is ever-evolving in humans and contributes to human prosperity. PIL/ikigai is related to the intrinsic and natural need for humans to establish a meaning for life, i.e., what makes life worth living, which every person ponders at some point of their life (Ishida, 2014; Ishida, 2016). 
In summary, the prevention and treatment of periodontitis and the enhancement of tooth tissue regeneration may depend on the accumulation of positive experiences. Therefore, in this study, the author proposes from the viewpoint of epigenetics that positive maternal attitudes and a positive lifestyle may contribute to the prevention and treatment of periodontitis and promote tooth tissue regeneration, leading to a higher QOL.

\section{Prefrontal Cortex, Neurotransmitters, and Hormones}

The PFC consists of a network of nerve cells, i.e., the neuronal network, and the electric signals in neurons and the chemical substances, called neurotransmitters, in synapses contribute to the communication of information (Ishida, 2016; Nolen-Hoeksema, Fredrickson, Loftus, \& Luts, 2014; Reece, Urry, Cain, Wasserman, Minorsky, \& Jackson, 2011). The PFC connects with other brain areas, such as those related to motor, exercise, visual, hearing, and memory functions (Ishida, 2016; Nolen-Hoeksema, Fredrickson, Loftus, \& Luts, 2014; Reece, Urry, Cain, Wasserman, Minorsky, \& Jackson, 2011). The functions of the PFC include the integration of information coming from other areas of the brain, planning, decision-making, inference and imaging, and the establishment of human relations (Ishida, 2016; Nolen-Hoeksema, Fredrickson, Loftus, \& Luts, 2014; Reece, Urry, Cain, Wasserman, Minorsky, \& Jackson, 2011). In addition, the maturity of a person depends on PFC function (Ishida, 2016; Nolen-Hoeksema, Fredrickson, Loftus, \& Luts, 2014; Reece, Urry, Cain, Wasserman, Minorsky, \& Jackson, 2011).

Cognition and emotion, which are associated with neurotransmitters and hormones, are indicators of welfare, i.e., positive emotions and cognition are associated with successes, while negative emotions and cognition are associated with failures in adapting to changing environments. In this paper, serotonin and melatonin will be discussed mainly in terms of their roles in positive and negative emotions and cognition. Serotonin is a neurotransmitter that is involved in homeostatic regulation and it facilitates prefrontal inhibition, i.e., serotonin modulates emotions by regulating the secretion of other neurotransmitters (Kabasawa, 2017; Ota, 2013). Serotonin is synthesized from tryptophan, which is an essential amino acid that is obtained from food, and secreted from various organs. Melatonin is involved in regulating the circadian rhythm, free radical scavenging, anti-oxidation, the immune system, anti-tumor activity, such as the inhibition of tumors, the generation of new vascular networks, DNA repair, body temperature, and bone induction activity, such as tooth development (Kabasawa, 2017; Obana-Koshino et al., 2015). Melatonin is secreted not only from the ovary, gastrointestinal tract, bone marrow, and lymphocytes, but also from the salivary gland (Obana-Koshino et al., 2015). Additionally, vitamins and minerals act as enzymes that enhance chemical responses (Ishida, 2016; Nolen-Hoeksema, Fredrickson, Loftus, \& Luts, 2014; Reece, Urry, Cain, Wasserman, Minorsky, \& Jackson, 2011). 


\section{Epigenetic Regulation by Serotonin and Melatonin}

Generally speaking, DNA methylation contributes to gene repression, while DNA demethylation contributes to gene expression (Obana-Koshino et al., 2015; Ota, 2013). Histone acetylation contributes to the transcription and translation of DNA (Obana-Koshino et al., 2015; Ota, 2013). As described in the Introduction section, epigenetic variations are inheritable and reversible (Kubota, 2016). In addition, epigenetic alterations are influenced by numerous other chemical reactions (Ota, 2013; Obana-Koshino et al., 2015).

\subsection{Serotonin and the Ability to Manage Stressful Events}

Serotonin can improve resistance to stress, the process of which may depend on the following chemical response (Ota, 2013). Increased levels of serotonin induce the expression of NGF-derived protein A, which decreases the level of DNA methylation on the promoter of the glucocorticoid (GR) receptor gene and promotes histone acetylation (Ota, 2013). As a result, GR receptor expression increases. GR is a steroid hormone that is secreted from the adrenal cortex. It is involved in the new production of sugar, and contributes to emotional stability. As a stress hormone, GR confers a higher resistance to stress. Decreased serotonin levels result in lower GR receptor levels, and contribute to decreasing stress tolerance (Ota, 2013).

\subsection{Melatonin and the Regeneration of Tooth Tissues}

Saliva contains melatonin, EGF, and NGF. Melatonin promotes bone induction activity, which induces mesenchymal stem cells to differentiate into bone-formation cells in places where bone does not exist (Kabasawa, 2017). Additionally, melatonin contributes to anti-aging mechanisms through the restoration of cranial nerves, which can help prevent or treat Alzheimer disease and dementia (Obana-Koshino et al., 2015). According to the functions and chemical structure of melatonin, melatonin may contribute to histone acetylation, which leads to the transcription and translation of genes (Yeh, Lin, Yang, Yang, Su, \& Yang, 2016). EGF is involved in regulating cell growth and proliferation, while NGF is involved in the extension of the axis cylinder of nerves, the induction of neurotransmitter synthesis, and the maintenance of nerve cell functions, i.e., restoration of cells after cell damage (Obana-Koshino et al., 2015). Therefore, saliva containing melatonin, EGF, and NGF may contribute to the regeneration of tooth bone, gingiva, and dental pulp.

\section{Experiences Influencing Positive Epigenetic Alterations}

Recent studies have shown that a well-balanced diet, which takes into consideration the importance of organic foods, moderate aerobic exercise, and PIL, can improve the ability of individuals to manage stressful events (Ishida, 2014). In contrast, an unbalanced diet, lack of exercise, and lack of PIL decrease the ability of individuals to manage stressful events. PIL, which reflects the function of the PFC, develops through warm-hearted human relations, successes with goals set 
by individuals themselves, and sufficient exposure to natural surroundings. Previous studies have indicated that individuals who experienced warm-hearted human contact with their mother as an infant had a better ability to manage stressful events as adults, while those with negative contact with their mother had a lower ability to manage stressful events (Ota, 2013). Positive maternal attitudes during infancy and a positive lifestyle are associated with the well-balanced synthesis and secretion of neurotransmitters, hormones, and vitamins. In particular, the sufficient secretion of serotonin and melatonin from not only the ovary, gastrointestinal tract, bone marrow, and lymphocytes, but also from the salivary gland may contribute to positive epigenetic alterations. In contrast, negative maternal attitudes and a negative lifestyle contribute to the unbalanced synthesis and secretion of neurotransmitters, hormones, and vitamins. As such, positive maternal attitudes and a positive lifestyle may help prevent periodontitis and promote the regeneration of tooth tissues. Nonetheless, habitual oral care is also important for preventing periodontitis.

\section{Limitations and Future Studies}

The limitations have not been clearly defined. Therefore, future studies are required to obtain more details and empirical evidence on the relation between experiences and epigenetic alterations, the roles of neurotransmitters, hormones, vitamins, minerals, and light catalysts, such as sunlight, and the effects of hydroxymethylcytosine on the process of DNA demethylation (Tajima, 2013).

\section{Conclusion}

Serotonin may contribute to a better ability to manage stressful events via growth factors. Saliva, the secretion of which is regulated by the autonomic nervous system, contains melatonin and growth factors, and melatonin may enhance the regeneration of tooth tissues with the help of various growth factors. Warm-hearted maternal attitudes during infancy and a positive lifestyle, which includes a well-balanced diet, moderate aerobic exercise, PIL, and other warm-hearted human relations, may increase the secretion of serotonin and melatonin in saliva. Increased secretion of serotonin and melatonin may contribute to a better ability to manage stressful events, and may enhance the prevention and treatment of periodontitis and the regeneration of tooth tissues.

\section{Contents of the Present Study}

The contents of the present study, including the relation between serotonin, melatonin, and improved stress tolerance, have been published in a different journal, Psychology (Ishida, 2016).

\section{Conflicts of Interest}

The author declares no conflicts of interest regarding the publication of this paper. 


\section{References}

Dhadse, P., Gattani, D., \& Mishra, R. (2010). The Link between Periodontal Disease and Cardiovascular Disease: How Far We Have Come in Last Two Decades? Journal of Indian Society of Periodontology, 14, 148-154. https://doi.org/10.4103/0972-124X.75908

Ferreira, M. C., Dias-Pereira, A. C., Branco-de-Almeida, L. S., Martins, C. C., \& Paiva, S. M. (2017). Impact of Periodontal Disease on Quality of Life: A Systematic Review. Journal of Periodontal Research, 52, 651-665. https://doi.org/10.1111/jre.12436

Ishida, R. (2014). Strategies for Promoting Health and Reducing Medical Experiences in Japan Using Purpose in Life/Ikigai, Moderate Exercise, and a Well-Balanced Diet. World Journal of Pharmacy and Pharmaceutical Sciences, 3, 257-274.

Ishida, R. (2016). Association between Purpose in Life/(Ikigai), Prefrontal Cortex Function, and the Prevention of Halitosis Caused by Mental Stress, Pseudo-halitosis, and Halitophobia. Psychology, 7, 271-274. https://doi.org/10.4236/psych.2016.73029

Kabasawa (2017). Nou wo Saitekikasureba Nouryoku wa 2 bai ni naru. Tokyo: Bunkyosha.

Kubota, T. (2016). Epigenetic Effect of Environmental Factors on Neurodevelopmental Disorders. Japanese Journal of Hygiene, 71, 200-207.

Martinez-Herrera, M., Silvestre-Rangil, J., \& Silvestre, F. J. (2017). Association between Obesity and Periodontal Disease: A Systematic Review of Epidemiological Studies and Controlled Clinical Trials. Medicina Oral, Patolog'́a Oraly Cirugía Bucal, 22, e708-e715. https://doi.org/10.4317/medoral.21786

Nolen-Hoeksema, S. N., Fredrickson, B. L., Loftus, G. R., \& Luts, C. (2014). Atkinson \& Hilgard's Introduction to Psychology (16th ed.). Stamford: Cengage Learning EMEA.

Obana-Koshino, A., Ono, H., Miura, J., Sakai, M., Uchida, H., Nakamura, W. et al. (2015). Melatonin Inhibits Embryonic Salivary Gland Branching Morphogenesis by Regulating Both Epithelial Cell Adhesion and Morphology. PLoS ONE, 10, e0119960. https://doi.org/10.1371/journal.pone.0119960

Ota, K. (2013). Epigenomu to Seimei. Tokyo: Kodansha (BLUE BACKS).

Reece, J. B., Urry, L. A., Cain, M. L., Wasserman, S. A., Minorsky, O. V., \& Jackson, R. B. (2011). Campbell Biology (9th Ed.). San Francisco, CA: Pearson Education, Inc.

Sakai, T. (2016). The Interaction between Saliva and Oral Function. JSPEN, 31, 675-680.

Sato, F., Tanaka, H., Saito, S., \& Chiba, M. (1998). The Purpose in Life Test. Tokyo: Shisutemu Pabulika.

Tajima, S. (2013). DNA Mechiruka no Seikagaku. In S. Tjima (Ed.), Epigenetics. Kyoto: Kagakudohjin.

Yeh, C. M., Lin, C. W., Yang, J. S., Yang, W. E., Su, S. C., \& Yang, S. F. (2016). Melatonin Inhibits TPA-induced Oral Cancer Cell Migration by Suppressing Matrix Metalloproteinase-9 Activation through the Histone Acetylation. Oncotarget, 7, 21952-21967. https://doi.org/10.18632/oncotarget.8009 